Objective To determine current seroepidemiology of CT infection in children in a US inner city population.

Design/methods Anonymized serum samples were obtained from children in 2 hospitals in Brooklyn, NY from 2012-2015. CT IgG was determined using EIA (Ani Labsystems). The following age strata were used: $11-12,13-14,15-16,17-18,19-20$ y.

Results 512 sera were included in the final analysis. Mean age 17 y. There were 192 (37.5\%) males and 320 (62.5\%) females. $\mathrm{CT}$ antibody was first detected at $16 \mathrm{y}$ and $18 \mathrm{y}$ for females and males, respectively. The prevalence per age-cohort were: Females: $11-14$ y-0, 15-16 y- 3.64\%, 17-18 y -15.9\%, 19-20 y $-14.75 \%$; Males: $11-16$ y- 0, 17-18 y- $8.51 \%, 18-20$ y- $9.33 \%$. Conclusions The prevalence of antibody was higher in girls than their male counterparts, mirroring national trends based on NAATs. Antibody was first detected in females at $16 \mathrm{y}$ and males at $17 \mathrm{y}$, reflecting sexual debut. Prior data from this cohort found antibody in $\%$ infants $<1 \mathrm{y}$, which disappeared between 1 and $16 \mathrm{y}$. The delay in male antibody detection may be due to later exposure and/or anatomical and physiological factors between the sexes. These data are critical in informing potential CT vaccine strategies. Future studies using a larger sample size and other populations will allow more precise estimates of age and gender-specific prevalence.

\section{P03.04 THE IMPACT OF UNIVERSAL CHLAMYDIA TRACHOMATIS (CT) SCREENING DURING PREGNANCY ON SEROEPIDEMIOLOGY OF CHLAMYDIAL INFECTION IN AMERICAN CHILDREN, 1991-2013}

N Banniettis, A Szigeti, S Sharma, K Chotikanatis, MR Hammerschlag*, S Kohlhoff. State University of NY Downstate Medical Center, Brooklyn, NY, USA

\subsection{6/sextrans-2015-052270.232}

Introduction CT remains the most prevalent sexually transmitted infection in developed and developing countries. Prenatal screening and treatment of pregnant women has resulted in a dramatic decrease of perinatal CT 1 infection (conjunctivitis, pneumonia) in the US. Before the implementation of screening, $\sim 50 \%$ of infants born to mothers with CT infection developed chlamydial conjunctivitis and/or pneumonia. However, there have been no studies of the incidence of perinatal CT infection, including seroepidemiologic studies, following the implementation of screening and treatment as recommended by the CDC in 1993.

Methods Anonymized banked serum and prospectively collected samples from children in Brooklyn, NY, were tested for CT IgG using the MIF assay. Serum samples were divided into 2 groups: 1: collected from 1991-1995, 2: from 2001-2013. Pts with C. pneumoniae (CP) infection (culture and/or antibody) were excluded.

Results 491 serum samples were identified (age range 0-20), 71 samples were excluded due to evidence of CP infection. 34\% of subjects $<10$ y in group 1 (pre-universal screening) had IgG against CT, while there were no positives in group 2 (post-universal screening), $\mathrm{p}<0.0001$. Children $>10 \mathrm{y}$ had a prevalence of $32 \%$ in group 1 and $3.48 \%$ in group $2, \mathrm{p}<0.0001$.

Conclusion Children $<10 \mathrm{yr}$ in group 1 (pre-screening) had relatively high rates of seropositivity, which were likely due to perinatal infection. This antibody was not due to $\mathrm{CP}$, as sera from children with CP infection were excluded. The significantly lower rates in group 2 (post-screening) confirm that prenatal screening and treatment of pregnant women has been effective for prevention of CT infection in infants. Persistence of antibody after perinatal infection may have implications for CT vaccine use in countries where prenatal screening and treatment has not been implemented.

\section{P03.05 CHLAMYDIA RE-TESTING AT SEXUAL HEALTH CLINICS HAS INCREASED BUT FURTHER INITIATIVES ARE NEEDED FOR YOUNG PEOPLE}

${ }^{1} \mathrm{~L}$ Watchirs Smith*, ${ }^{1} \mathrm{D}$ Callander, ${ }^{1} \mathrm{H}$ Ali, ${ }^{1,2} \mathrm{C}$ Bourne, C O'Connor 1,3,4, ${ }^{5} \mathrm{D}$ Lewis, ${ }^{6} \mathrm{~B}$ Forrester, ${ }^{7} \mathrm{~V}$ Hounsfield, ${ }^{1} \mathrm{~J}$ Kaldor, ${ }^{8} \mathrm{M}$ Hellard, ${ }^{1} \mathrm{~B}$ Donovan, ${ }^{1} \mathrm{R}$ Guy, On behalf of the ACCESS collaboration. 'Kirby Institute, UNSW Australia, Sydney, Australia; '2Sydney Sexual Health Centre, Sydney, Australia; ${ }^{3}$ Sexual Health Service, Community Health, Sydney Local Health District, Australia; ${ }^{4}$ Central Clinical School, Sydney University, Australia; ${ }^{5}$ Western Sydney Sexual Health Centre, Sydney, Australia; ${ }^{6}$ Barwon Health, Geelong, Australia; ${ }^{7}$ North Shore Sexual Health Service, Sydney, Australia; ${ }^{8}$ Burnet Institute, Melbourne, Australia

\subsection{6/sextrans-2015-052270.233}

Introduction Chlamydia is the most commonly notified infection in Australia; most diagnoses are in young people, and re-infections are common. Re-infection leads to onward transmission and increases the risk of reproductive morbidity and HIV transmission. Guidelines recommend re-testing 3 months following treatment to detect re-infections. We assessed trends in re-testing after a chlamydia diagnosis in Sexual Health Clinics (SHCs) in New South Wales (NSW) over a 5 -year period and factors associated with re-testing.

Methods Routine patient data from 2009 to 2013 were extracted from 33 SHCs. A Chi-2 test was used to assess time trends in the proportion re-tested in $2-4$ months following a chlamydia diagnosis and also 2-12 months, in a range of risk groups. Multivariate logistic regression was used to determine demographic, risk behaviour and clinic factors associated with re-testing at 2-4 months, adjusting for clinic clustering.

Results Overall 8,646, chlamydia diagnoses were analysed and $1,281(15 \%)$ were re-tested in $2-4$ months (23\% of GBM, $25 \%$ of sex workers, $12 \%$ of young heterosexuals aged $<30$ years), with a significant increase over time $(13 \%$ in 2009 to $18 \%$ in 2012, $\mathrm{p}<0.01)$. In a broader time frame of $2-12$ months, retesting was higher at $26 \%$ ( $42 \%$ of GBM, $41 \%$ of sex workers, $20 \%$ of young heterosexuals) with a modest increase over time ( $25 \%$ to $30 \%, \mathrm{p}<0.01)$. Factors associated with re-testing in 24 months were: being GBM (adjusted odds ratio $(\mathrm{aOR})=1.65$, $95 \% \mathrm{CI}: 1.44-1.90, \mathrm{p}<0.01)$, current sex work $(\mathrm{aOR}=$ 2.04,95\% CI: $1.65-2.52, \mathrm{p}<0.01$ ), attending the clinic $>5$ times (aOR: 3.11,95\% CI: 2.62-3.70, p < 0.01) and people attending clinics with SMS reminders $(\mathrm{aOR}=2.25,95 \% \mathrm{CI}$ : 1.16-4.37, $\mathrm{p}=0.01)$.

Conclusions Re-testing at 2-4 months after a chlamydia diagnosis increased over time, but remains low. GBM and sex workers were more likely to be re-tested, perhaps because they were attending anyway. Attending clinics with SMS reminders increased the likelihood of re-testing. Additional strategies, such as home-collection, may be needed to increase re-testing in young heterosexuals.

Disclosure of interest statement The ACCESS Sexual Health Services Network is funded by the NSW Ministry of Health, Victorian Department of Health, Australian Capital Territory Department of Health, and the Northern Territory Department of Health. 


\section{Abstracts}

\section{P03.06 SEXUAL EXCHANGES AND COERCION AMONG UNDERGRADUATE STUDENTS IN NIGERIA: IMPLICATIONS FOR SEXUALLY TRANSMITTED INFECTIONS}

Adeyemi Oluwagbemiga*. Federal University Oye-Ekiti

$10.1136 /$ sextrans-2015-052270.234

Background Numerous studies have been documented in Nigeria to assess the magnitude of sexual coercion there is limited literature that linked sexual coercion with sexual exchanges, and vulnerability among students. This study therefore examines the relationship between sexual exchanges and coercion with its implication on Sexually Transmitted Infections (STI) among undergraduate students in the study area.

Methods A multi-stage random sampling procedure was employed in administration of 1458 questionnaires. Three levels of analysis were used for this study.

Results From the survey, half of the respondents had their first sexual experiences before age 16 years while $40 \%$ of the respondents had sexual intercourse with two or more partners in the last six months preceding the survey. Two-thirds of the respondents did not use condom in their last sexual intercourse. The study established a significant relationship between sexual coercion and ever had STIs, period of relationship, use of condom, having multiple partners and STI treatment behaviour. While the logistic regression shows that respondents aged 15-19 years are 2.568 times more likely to be coerced $\left(\mathrm{P}\right.$ value $\mathrm{X}^{2}=$ 0.00 CI 1.717-3.839) when compared with the reference category aged 20-24 years. There is a positive relationship between sexual coercion and sexual gratification; those who received gifts (both materials and money) are 1.947 times more likely to experience sexual coercion when compared with those who receive nothing.

Conclusion and recommendation Violence limits women's ability to manage their reproductive health and exposes them to sexually transmitted diseases. There is need for holistic approach to the issue of sexual coercion especially among young females based on the health consequences and the psychological trauma for the experience they did not prepare for. There is need for policy action and health education against sexual coercion in the study area.

\section{P03.07 MITIGATING THE IMPACT OF HIVIAIDS AMONG IN AND OUT OF SCHOOL YOUTH THROUGH PEER EDUCATION USING FAMILY LIFE HIV EDUCATION IN FEDERAL CAPITAL TERRITORY ABUJA NIGERIA}

Falilu Opeyemi Agbaje*, Farinloye Makanjuola. OROL Youth Empowerment Initiative

\subsection{6/sextrans-2015-052270.235}

HIV AIDS has systematically permeated the entire Nigerian social fabric, affecting males and females in both urban and rural areas, as well as adolescent young people.

This paper presents findings from an evaluation of a HIV prevention program designed to determine the effects of HIV prevention intervention (HPI) on in school youths in AMAM Local Government Area Abuja Nigeria.

Data were collected from 75 trained peer educators from 3 Schools in AMAC Local Government Area FCT Abuja Nigeria and also 100 in school youths who were not trained. Data were also collected at the beginning and end of the intervention among 950 in school youths that were reached with peer education in 3 Schools and also among other 1100 in school youths from 3 other schools that were not reached with the program using qualitative and quantitative research methods.

The findings revealed that the programme had several positive effects on the students such as increase in knowledge of HIV/AIDS, adoption of preventive behaviour and acquisition of life skills. The quantitative data shows the knowledge of the respondents have increased by $71 \%$ at the end of the intervention and the result also shows that the students trained as peer educators have higher knowledge of HIV prevention and life skills than those who were not trained.

The quantitative and qualitative data show that the project has produced several positive multiplier effects on the knowledge and behaviour of youths. The findings also show It is necessary to focus on young people because they are at the centre of the HIV/AIDS epidemic. The project shows that Peer education is one of the best approaches to providing comprehensive knowledge on HIV/AIDS/STIs and related issues, as it provide an excellent environment for effective peer-to-peer learning. There is therefore the need to sustain and even expand the program such that we can reach out to other youths in other areas of the city and even other states of the country.

\section{P03.08 PREVAILING MYTHS AND MISCONCEPTIONS AND EFFECT OF INTERVENTION IN CHANGING THE ATTITUDE OF SECONDARY SCHOOL STUDENTS OF SOUTH DELHI REGARDING HIVIAIDS AND POSITIVE PERSONS}

SK Badhan*, Rani Usha, Badhan Sonal, SN Ojha. HKMC Trust, Sarita Vihar, New Delhi

\subsection{6/sextrans-2015-052270.236}

Background Despite detailed information available regarding HIV infection, a lot of misconceptions and the infection is still regarded as a taboo and the positive person is discriminated. Youth can play an important role in removing this taboo.

Methods The NGO is involved in giving life skills education to school children. This study part of the same involved students of class IX onwards selected from eight schools of South Delhi.

Students were administered anonymous semi structured pre tested pro forma seeking information regarding age, sex, various myths/misconceptions regarding HIV infection and their attitude towards HIV positive person. After a detailed brainstorming interactive session the students were given one hour break and they were asked to visit the hall where IEC material in the form of leaflets and charts was displayed. After the break same pro forma marked ' $\mathrm{B}$ ' was given to be filled.

Results A total of 400 students were given the pro forma and there were 8 refusals, giving a response rate of $98 \%$. Of these 192 males and 200 females responded. Myths/misconceptions regarding transmission were: through blood donation 154 (39.28\%), swimming in common pool 225 (57.39\%), playing together $278(70.91 \%)$ and shaking hands $202(51.53 \%)$ and after intervention came down respectively to 54 (13.77\%), 98 (25.0\%), 95 (24.23\%) and 89 (22.70\%).

Attitude towards a positive person was adjudged by asking fear of eating 276 (70.4\%), having friendship 191 (48.7\%), allowed to attend school $270(68.9 \%)$ and by taking care 319 (81.4\%), and the same changed respectively after intervention to: 116 (29.59\%), 331 (84.43\%), 352 (89.79\%) and 376 (95.91\%). 